\title{
L'universo femminile, lo spazio domestico e la famiglia nelle opere di Clara Sereni
}

\author{
Elis Deghenghi Olujić \\ Università Juraj Dobrila di Pola \\ Dipartimento di studi in lingua italiana
}

\section{Riassunto}

Nell'intervento si evidenziano alcuni aspetti della narrativa di Clara Sereni, prendendo in esame soprattutto due lavori: Casalinghitudine (1987) e Il gioco dei regni (1993). Il punto d'avvio per delineare il profilo dell'autrice è invece il Taccuino di un'ultimista (1998), nel quale la Sereni, a partire dalla sua molteplice identità, di donna, ebrea, comunista e madre "handicappata", propone una sfida: non cancellare la diversità, ma accettarla e farne una risorsa utile ai fini della conoscenza degli altri e di se stessi. Inoltre, con l'analisi del romanzo genealogico Il gioco dei regni, il lavoro si propone di dare il giusto rilievo alla "diaspora affettiva" della Sereni, che ritorna nei luoghi dei suoi avi per rintracciare tutti quei fili dispersi che, tessuti insieme, hanno dato vita a uno dei più bei "libri di famiglia" scritti in Italia negli ultimi anni. Il contributo esamina i personaggi e i cronotopi sereniani, evidenzia le caratteristiche della scrittura dell'autrice romana che tra femminilità, ebraismo e politica, è anche la ricerca di un ordine della memoria, un dialogo con figure storiche appartenenti a generazioni diverse, una presa in carico della quotidianità e della storia e insieme itinerario intellettuale.

Parole chiave: memoria, storia, cibo, ricetta, famiglia, utopia 


\title{
1. Il Taccuino di un'ultimista, ossia della diversità e dell'utopia
}

\author{
Soltanto il bisogno cosi umano di comunicare può \\ portarci ad affrontare la vicenda mai per sempre \\ risolta della scrittura.
}

(Clara Sereni, Taccuino di un'ultimista, 1998)

Non è compito facile presentare Clara Sereni, che occupa un posto ben riconoscibile nella narrativa italiana contemporanea. Tentiamo di farlo con i dati bibliografici che di seguito riportiamo e con il sostegno del Taccuino di un'ultimista, il diario pubblico e privato dell'autrice che si racconta al meglio nelle sue opere, nelle quali percorsi ed esperienze della vita privata e professionale s'intrecciano indissolubilmente.

Narratrice, editorialista, da decenni impegnata in politica e nel mondo associazionistico, la Sereni è nata a Roma nel 1946, ed ha iniziato a scrivere negli anni Settanta dello scorso secolo. Perugina d'adozione, nel capoluogo umbro ha ricoperto dal 1995 al 1997 la carica di vicesindaco e di assessore alle politiche sociali, un'esperienza che l'ha particolarmente coinvolta e che ha risucchiato tutte le sue energie, al punto da frantumare gli equilibri familiari e alterare le relazioni sociali. La politica è una piovra dai mille tentacoli, che impedisce di mantenere una parvenza di vita privata. Questa la conclusione cui si perviene dopo aver letto Passami il sale (2002), dove con stile rigorosamente semplice, che connota tutta la sua scrittura, la Sereni racconta lo snodarsi della vita dal momento in cui ha accettato di ricoprire una carica amministrativa per incidere con caparbietà sulla qualità della vita nella propria città, per testimoniare la propria lotta contro le barriere dell'indifferenza nei luoghi dove queste barriere possono essere abbattute. Una scelta impegnativa, specie per una donna sempre scissa tra riunioni di giunta e il bucato da stendere tanto che, confessa, se un'amica le chiedesse un parere rispetto a un'ipotesi di entrare in politica, per il suo bene la sconsiglierebbe di farlo. È difficile per una donna conciliare i diversi oneri lavorativi e familiari, sottrarre tempo agli affetti. Nel caso della Sereni si è trattato di una scelta coraggiosa, determinata dal desiderio di restituire qualcosa di ciò che si è avuto in sorte operando per la realizzazione di ideali granitici in cui si crede, e forse, come qualcuno ha ipotizzato, un tributo ideale al lavoro politico del padre Emilio, esponente di spicco del PCI togliattiano ${ }^{[1]}$.

Clara Sereni ha esordito come scrittrice nel 1974 con Sigma Epsilon, una rivisitazione in chiave autobiografica del frenetico impegno politico che ha caratterizzato la sua generazione, quella che ha le proprie radici nel Sessantotto, un'epoca che la Sereni considera costitutiva. La prima esperienza letteraria è stata fallimentare.

\footnotetext{
[1] Emilio Sereni, antifascista della prima ora, studente della facoltà di Agraria a Portici, vicino Napoli, ha aderito nei primi anni del fascismo al Partito Comunista Italiano, ricoprendo subito una funzione dirigenziale. Dopo la caduta del fascismo è stato deputato del P. C. I. e ha fatto parte del primo governo democratico come ministro del lavoro. Sereni appartiene a quella generazione di comunisti con una grande carica morale ed etica, la cui formazione era avvenuta in un partito che si muoveva negli anni difficili del fascismo all'ombra dell'Unione Sovietica, un dirigente ortodosso la cui fedeltà alla linea del Partito non fu messa in discussione né in occasione dell'invasione dell'Ungheria del 1956, né dalla Primavera di Praga, né dagli attacchi della nuova sinistra che non gli venivano risparmiati neanche in casa, come la Sereni ricorda soprattutto in Casalinghitudine. Quando si tratterà di dare l'avvio a un processo di rinnovamento e riforma, da personaggi come lui il Partito prenderà velocemente le distanze. Emilio Sereni fu una vittima di questo rinnovamento. Rimase chiuso in una rete di doveri e rigidezze. Ne Il gioco dei regni, Clara Sereni ricostruisce la figura del padre recuperando "tra i detriti della Storia" quello che la Storia non registra, ovvero quei tasselli che permettono di restituire carne e sangue a figure come quella di Emilio Sereni, troppo spesso appiattite dagli stereotipi della lotta politica. Con una narrazione puntellata da documenti, brani di libri, lettere e diari, pubblici e non, ne Il gioco dei regni la Sereni va alla ricerca delle ragioni più profonde del rigore paterno, che non rimaneva confinato al campo della politica, ma impregnava di sé finanche la vita di tutti i giorni e i rapporti in seno alla famiglia.
} 
L'autrice la definisce "bruciante"[2]. L'opera fu difatti accolta dal silenzio della critica e dall'incomprensione del suo compagno, che provava una malcelata gelosia verso l'atto di autonomia rappresentato dalla scrittura ${ }^{[3]}$. Di seguito, per oltre dieci anni, si è dedicata intensamente alle traduzioni di Stendhal, Balzac e Madame de Lafayette. Solo nel 1987 è tornata alla narrativa con Casalinghitudine, che l'ha fatta conoscere al grande pubblico e anche all'estero. Nel 1989 ha pubblicato Manicomio primavera, un'opera nella quale, sotto un titolo ispirato a una struggente lirica della poetessa Sylvia Plath, ha raccolto tredici strazianti racconti brevi, nei quali oltrepassa la tenue linea di demarcazione tra salute e malattia, normalità e follia, benessere e disperazione. Gli ultimi sette racconti sono idealmente legati tra loro, sono brani di una stessa vita: quella di una madre alle prese con l'handicap del proprio figlio, una condizione che la Sereni ha sperimentato in prima persona, perché madre di Matteo, un figlio con problemi psichici. Non stupisce pertanto che il suo impegno, oltre che alla letteratura e alla politica, sia da sempre rivolto al campo sociale. Nel 1998 ha promosso la fondazione della Città del Sole, impegnata nella realizzazione di progetti di vita per persone con disabilità psichiche e mentali, di cui riveste il ruolo di presidente. Nel 2004, insieme al marito, il regista Stefano Rulli, ha girato un documentario intitolato Un silenzio particolare, rappresentato alla Mostra del cinema di Venezia, che racconta il disagio mentale del figlio e il modo con cui l'hanno affrontato come genitori, senza piangersi addosso. In questo caso le immagini hanno la forza che le parole non riescono mai ad avere, e raccontano una piccola utopia concreta, ossia la capacità di Matteo di imparare a vivere e di raggiungere un minimo di autonomia, nonostante il disagio mentale. Ė il sogno (o l'utopia) della Sereni: promuovere una cultura del cambiamento a favore dell'integrazione, per offrire ai malati concrete opportunità di interazione con la società, che deve essere preparata e disposta ad accoglierli senza pietismo ed assistenzialismo, ma con la consapevolezza del contributo che essi possono apportare. Crescita civile e solidarietà sono i punti fermi di quest'utopia, nella quale la Sereni continua a credere perché non sbiadisca nel tempo.

La fama di scrittrice della Sereni accresce con il romanzo Il gioco dei regni (1993), ristampato nel 2007 con la pregnante Prefazione di Alberto Asor Rosa. Vanno ancora annoverate, in ordine cronologico, Eppure (1995), che raccoglie undici racconti brevi, tracciati a tratti lievi, che illuminano per un momento, fissandola, la tragedia della normalità: i lavori collettivi Mi riguarda (1994) e Si può (1996), pubblicato quest'ultimo nell'anno in cui è diventata obbligatoria la chiusura definitiva di quel che restava degli ospedali psichiatrici, a quasi quarant'anni dall'entrata in vigore della Legge Basaglia. È del 1998 Taccuino di un'ultimista, un libro di appunti che raccoglie alcuni interventi scritti per quotidiani e convegni, nel quale la scrittrice sviscera ogni componente emotiva e

[2] A proposito dell'opera d'esordio a pp. 37 e 38 del Taccuino di un'ultimista la Sereni confessa: “[...] un brutto libro, al quale sono tuttora affezionata. Sigma Epsilon era tanto brutto perché - mi si scusi la presunzione - era un figlio nato settimino, un libro in anticipo di sei mesi o un anno rispetto all'abbondante messe di libri di donne, belli e brutti, che poi uscirono. Parlo dei libri in cui le donne cominciavano a riflettere sulla propria autobiografia: in genere in tono lamentoso (anch'io non facevo eccezione), ma compiendo una tappa importante del loro cammino di coscienza e crescita".

[3] A p. 129 de La nuova narrativa italiana. Travestimenti e stili di fine secolo (Bollati Boringhieri, Torino, 1999), scrivendo a proposito de Il gioco dei regni, Filippo la Porta evidenzia, a ragione, che "[...] l'unico valore coltivato dalla famiglia Sereni è la scrittura, [...] con la quale tutti i personaggi stabiliscono un rapporto intenso, vorace". Non stupisce pertanto che la Sereni abbia considerato da sempre la scrittura come un privilegio, coltivato con passione fin da bambina. Nel rilevare il valore che tutti i Sereni attribuivano alla scrittura, a pp. 56-57 de Il gioco dei regni l'autrice annota: "Preoccupati della traccia che avrebbero lasciato, inconsapevoli a lungo del riannodarsi di una tradizione ancestrale, si garantirono con la scrittura la sopravvivenza, si affidarono alla carta per capire e farsi intendere, per rendere leggibile - dunque accettabile - ogni cosa: le fantasie dell'infanzia come le sofferenze ancora inconsapevoli”. 
psicologica che ha accompagnato e costellato la sua vita di successi, delusioni e sconfitte, come la forzata dimissione dalla carica politica, dalla quale fu espulsa perché col suo fare poco accomodante e per nulla accondiscendente, scuoteva consolidati assetti di potere maschile. Fanno seguito Da un grigio all'altro (1998), Passami il sale (2002), Le merendanze (2004) e Il lupo mercante (2007).

La Sereni, oltre che come scrittrice, è nota per il suo impegno politico e civile, sempre contraddistinto da una forte carica utopica e dalla volontà di valorizzare tutte le differenze: quelle di genere, quelle etniche, religiose, politiche, fino a quelle che nascono dalla malattia, fisica e mentale. L'opera che consente di conoscere meglio l'autrice, che ha sperimentato sulla propria pelle diversità tutte ugualmente scomode e spesso difficilmente conciliabili fra loro, è Taccuino di un'ultimista (1998). Si tratta del resoconto di un percorso di maturazione individuale e collettiva, ma soprattutto è il diario di una donna che s'interroga con disarmante sincerità sulla sua molteplice identità: di donna, di ebrea, d'intellettuale, di comunista e madre "handicappata". I brani sono organizzati in quattro sezioni: Shalom, L'agenda dei desideri, Benvenuto e La partita truccata. Calendario di un conflitto, corrispondenti ai quattro "spicchi" in cui si articola la sua identità, fatta come "un mosaico di tessere mal tagliate" ${ }^{[4]}$. A prima vista ogni brano appare concluso in se stesso, legato com'è a un sentimento, un episodio, un fatto di cronaca, un'emozione. Ogni episodio risuona nell'animo della scrittrice con una nota personale, ed è così che lei ce lo consegna, segnato da una soggettività che lo sottrae alla nuda cronaca e lo integra in un discorso unitario, sempre in bilico tra testimonianza, riflessione e letteratura. Nell'Introduzione, spiegando le parti in cui si suddivide l'opera, scrive:

Non fingo alle quattro partizioni di questo libro un'oggettività esterna, ma le dichiaro come i quattro spicchi dei quali, con continui sconfinamenti, mi sembra di compormi: ebrea per scelta più che per destino, donna non solo per l'anagrafe, esperta di handicap e debolezze come chiunque ne faccia l'esperienza, utopista come chi, radicandosi in quanto esiste qui e oggi, senza esimersi dall'intervenire sulla realtà quotidiana coltiva il bisogno di darsi un respiro e una passione agganciati al domani ${ }^{[5]}$.

Nonostante la fatica di dare coerenza a queste parti di sé e farle stare insieme, la Sereni propone una sfida: non azzerare la diversità, ma accettarla insieme ai conflitti che essa provoca, e farne una risorsa utile ai fini della conoscenza di se stessi e degli altri, lo strumento per impedire che ogni singola persona e il mondo vadano in pezzi. Per la Sereni la sfida è dunque riuscire a tenere insieme tutte le parti che la compongono, perché annullare o anche solo ignorare una, significherebbe perdere una parte di sé, significherebbe perdere parte di quella diversità che è portatrice di ricchezza ${ }^{[6]}$. Non stupisce pertanto che la sua scrittura sia tutta caratterizzata dallo sforzo e dalla capacità di connettere e armonizzare, senza annullarle, le differenze e le distanze: la storia personale e quella di una generazione, la dimensione domestica e il mondo esterno, la memoria

[4] La metafora, con numerose varianti, appare più volte nei testi della Sereni. La troviamo riportata per la prima volta in Casalinghitudine (1987).

[5] Sereni, C., Taccuino di un'ultimista, Feltrinelli, Milano, 1998, pp. 11-12.

[6] Particolarmente difficile è rivendicare l'appartenenza insieme all'ebraismo e alla schiera di coloro che si definiscono comunisti. Si veda a tale proposito l'articolo della Sereni apparso sul quotidiano "l'Unità" il 16 gennaio 2006 con il titolo "La colpa di essere ebrea", che ha suscitato non poche polemiche. Alla riflessione sulla malattia mentale e su altre dolorose diversità sono dedicate due raccolte di racconti brevi, Manicomio primavera e Eppure. Si vedano anche i molti interventi della scrittrice sulla stampa quotidiana e le due opere collettive Mi riguarda (che contiene Diario, la testimonianza della sua vicenda personale) e Si può. 
privata e la memoria collettiva. In tutte le sue opere, spesso con grande originalità, la Sereni s'interroga sulle differenze e sull'identità, che costruisce costantemente e con pazienza, giorno dopo giorno. Perché l'identità non si presenta mai monolitica, non si basa sull'affermazione di un insieme di valori precostituiti bensì è esercizio ed esperienza, esplorazione continua e superamento dei confini, curiosità creativa, lotta per l'autonomia.

Nell'illuminante Introduzione di Taccuino di un'ultimista la Sereni spiega anche il suo rapporto con la scrittura, e rivela al contempo il metodo seguito nella stesura di questo diario discontinuo, costituito da brani e articoli apparsi su giornali e riviste, o anche inediti, che coprono un arco di circa dieci anni. A p. 7 scrive:

Insegnanti delle scuole primarie e psicopedagogisti sanno bene, però, quale grande avventura presupponga e incarni l'atto dello scrivere: una rappresentazione di sé che ha bisogno di molta fiducia per affidarsi - con un atto che è del corpo ma mette in gioco l'anima - al segno e alla carta. Segno e carta in grado di vivere un'esistenza autonoma, fuori di ogni protezione e controllo: ogni lettera tracciata è un pezzettino di sé libero di andarsene in giro per il mondo in totale autonomia, esposto a rischi e fortune incontrollabili. [...] Soltanto il bisogno così umano di comunicare può portarci ad affrontare la vicenda mai per sempre risolta della scrittura ${ }^{[7]}$.

Inoltre, per spiegare il senso della faticosa operazione di recupero messa in atto nell'opera e raccontare il percorso seguito nella riappropriazione complicata di articoli e brani già pubblicati, dichiara: "[...] il percorso verso questo titolo e questa copertina non poteva esaurirsi nell'operazione - relativamente semplice - di giustapporre i pezzi sparsi, ma doveva riuscire a ridare continuità e senso a un materiale discontinuo e disorganico. Insomma, dovevo tornare a riconoscere come mie parti delle quali - nel momento in cui le avevo date alle stampe - avevo ritenuto di potermi liberare" ${ }^{[8]}$. Un primo importante gesto di riappropriazione e assunzione di responsabilità, confessa l'autrice, "[...] è stato intercalare quella che avevo raccontato a me stessa e agli altri come una scrittura totalmente pubblica con dei piccoli inediti, i sussulti di una scrittura "mia" registrati in due anni di sovraesposizione, superlavoro, spossessamento di ruolo e identità" ${ }^{[9]}$. Le dichiarazioni qui riportate consentono di concludere che l'opera si presenta come un itinerario intellettuale che, per quanto "spurio e zigzagante" (così lo definisce la Sereni a p. 36), tra femminilità, ebraismo e politica, rappresenta il tentativo di dare un ordine alla memoria e sostenere la necessità della presa in carico, da parte della scrittura, della quotidianità e della storia.

La ragione che ha spinto la Sereni a scrivere è dunque molto semplice, perché insita nella natura umana: l'atto dello scrivere è sempre connesso a un voler mettere ordine nell'esistenza e nei pensieri. È così anche per la Sereni, che nella scelta di scrivere vede "[...] la sensazione magica di poter dare ordine, con le parole, al mondo: un mondo che nell'allinearsi delle righe, nell'accumularsi delle pagine, nel contenimento garantito dalla copertina trova momentaneamente una comprensibilità, e la coerenza che alla vita non appartiene" ${ }^{[10]}$. Con la scrittura si soddisfa l'esigenza tanto umana di comunicare

[7] Sereni, C., Taccuino di un'ultimista, op. cit., p. 7.

[8] Ivi, p. 9.

[9] Ivi, p. 11. I due anni cui l'autrice fa riferimento sono quelli intercorsi tra il 1995 e il 1997, durante i quali ha ricoperto la carica di vicesindaco di Perugia.

[10] Ivi, p. 8. 
un'esperienza, di condividerla con gli altri, si dà voce a un bisogno intellettuale insopprimibile, che non si riesce e non si vuole arginare. Per la Sereni la scrittura è "un'operazione di definizione, di mediazione", un gesto di "autonomia profonda" ${ }^{[11]}$. Essa rappresenta il suo secondo "privilegio", essendo il primo quello d'essere nata e cresciuta in una famiglia di donne, "formata da una nonna, una prozia, due sorelle maggiori di me, una madre e poi un'altra, e niente figli maschi”"[12]. Questa famiglia particolare ed emblematica, dove il padre era "molto presente in termini psicologici, ma sostanzialmente assente dalla vita quotidiana" ${ }^{[13]}$, ha fatto sì che l'autrice considerasse l'essere donna una cosa normale, che le dava parità di diritti. Crescere in un ambiente nel quale dominavano le presenze femminili, le ha permesso di crescere libera da qualsiasi "tipo di stereotipo ritardante o frenante" ${ }^{[14]}$, per cui essere donna non era considerato né un valore né un disvalore, ma "qualcosa cui guardare con attenzione prudente, per non cadere nelle trappole di una femminilità connotata da fronzoli e smancerie" ${ }^{\text {[15]. }}$.

La scrittura richiede tempo soprattutto per sé, un tempo di cui le donne, per le dispersive incombenze domestiche e familiari, non possono disporre liberamente. Ecco che allora il gesto di scrivere diventa quasi trasgressivo, perché sottrae la donna al cosiddetto "ordine naturale" in cui essa è custode di una domestica liturgia, prolungamento della materia stessa, in contrasto con quel di spirituale e distaccato, così pertinente alla scrittura. Per troppo tempo esclusa dai luoghi di creazione del sapere, la donna ha elaborato nel tempo un pensiero assai diverso da quello maschile, perché molto più vicino alle problematiche della vita quotidiana che all'astrazione teorica. Come è stato spesso ripetuto, il sapere femminile, piuttosto che l'intelletto, predilige ascoltare i suggerimenti di una sensibilità legata al corpo, che dà la vita e nutre. C'è chi reputa che parlare di "scrittura femminile" sia in larga misura abusivo, perché "maschile" e "femminile" sono concetti di sfuggente definizione e si scambiano sempre di più le parti nei singoli autori, indipendentemente dal sesso di appartenenza ${ }^{[16]}$. Ma nel caso della Sereni pare lecito parlare di "scrittura femminile", in primo luogo perché la sua narrativa smentisce il preconcetto che la scrittura femminile non sia innovativa sul piano delle strategie narrative, e in secondo luogo perché in essa si avverte un'evidente diversità (nel rapporto con la scrittura appunto e con il mondo), che ne costituisce la cifra più autentica. Una naturale contiguità di termini solitamente contrapposti: da un lato la quotidianità e un senso molto fisico, quasi tattile della realtà, al quale si

[11] Sereni, C., "Scrivere per non mangiarsi il cuore", in Conversazioni di fine secolo, Iaia Caputo e Laura Lepri (a cura di), La Tartaruga, Milano, 1995, p. 171.

[12] Sereni, C., Taccuino di un'ultimista, op. cit., p. 36.

[13] Ibidem.

[14] Ibidem.

[15] Ivi, p. 49

[16] Nello specifico si fa riferimento a quanto sostenuto da Filippo La Porta a p. 115 del capitolo intitolato Dove porta la mente, inserito in La nuova narrativa italiana. Travestimenti e stili di fine secolo, Bollati Boringhieri, Torino, 1999. Pur mettendo in forse la legittimità della "scrittura femminile", La Porta dedica un capitolo ad alcune autrici italiane contemporanee. Risultano nella lista: Susanna Tamaro, Fabrizia Ramondino, Paola Capriolo, Clara Sereni, Sandra Petrignani, Marisa Volpi, Cristina Comencini, Grazia Livi, Elena Gianini Belotti, Lalla Romano e Luce D'Eramo. Prendendo come spunto il titolo del best-seller della Tamaro, nel capitolo il critico afferma che idee e passioni (ovvero cuore e mente) possono stare insieme ed essere tra loro intrecciate. Perché, conclude, la "migliore narrativa femminile di questi anni racconta i sentimenti in modo antisentimentale (si pensi ai racconti di Sandra Petrignani o di Valeria Viganò), così come mette alla prova le idee attraverso un cortocircuito con la vita affettiva (Clara Sereni)". Tra le scrittrici, Natalia Ginzburg è stata colei che si è fermamente opposta alla differenziazione degli scrittori sulla base del sesso, invocando il diritto d'essere considerata scrittore e non scrittrice. Inoltre, non amava le raccolte di opere esclusivamente femminili, in quanto temeva che questo portasse ad una marginalizzazione della produzione letteraria delle donne. Ribadì questa opinione molto chiaramente in un'intervista rilasciata a Mary Gordon e pubblicata il 25 marzo del $1990 \mathrm{sul} \mathrm{"New} \mathrm{York} \mathrm{Times} \mathrm{Magazine"} \mathrm{(p.} \mathrm{42),} \mathrm{in} \mathrm{cui} \mathrm{dichiarò} \mathrm{che} \mathrm{uno} \mathrm{scrittore} \mathrm{era} \mathrm{semplicemente} \mathrm{uno} \mathrm{scrittore:} \mathrm{quello} \mathrm{che}$ importava era lo scrivere non l'essere uomini o donne. 
aggiunge un rapporto disarmato e di trasparenza con la verità, e dall'altro l'attrazione per l'utopia ${ }^{[17]}$. La definizione di "femminile" per la scrittura della Sereni è giustificata anche dal fatto che è l'autrice stessa a spiegare il suo rapporto (molto femminile) con la pratica scrittoria. Racconta che quando annunciò al suo compagno, di riconosciuta $\mathrm{e}$ indubbia qualità intellettuale, il desiderio di tornare a scrivere dopo anni di silenzio, un ritorno che si è rivelato difficile e tortuoso, lui le raccomandò di prepararsi, le consigliò una bibliografia ragionata di tutto quello che, a suo parere, doveva leggere per affrontare e sviluppare l'idea che aveva in mente. L'autrice si rifiutò di applicare il metodo che le era stato suggerito. Rispose che avrebbe letto dopo, ben determinata a rivendicare "un mio modo (femminile!) di lavorare, diverso dal suo (maschile)" ${ }^{[18]}$. È stata una scelta necessaria e lungimirante, che si è rivelata giusta. In definitiva, significava affermare il valore dell'intuizione rispetto alla sintesi, anche se, ammette la Sereni, "allora non ero minimamente in grado di parlare in questi termini, né tanto meno di rivendicare come valore una specificità di genere" ${ }^{[19]}$.

Sembra d'obbligo a questo punto inserire un brano in cui l'autrice spiega l'atteggiamento verso la scrittura, e quella femminile in particolare, ritenuta un'araba fenice tuttora denegata da molti, sostenuta da altri, ma ancora poco riconosciuta, anche se ci sono di certo scritture che fin dalle prime righe denunciano la loro appartenenza di genere. A conclusione di un articolo in cui riflette sul privilegio rappresentato dalla pratica scrittoria, dopo aver ringraziato le donne, che con il loro consenso l'hanno fatta sentire più autorizzata e "in diritto" di compiere "quella scalata al cielo, al potere, che è la scrittura" ${ }^{[20]}$, la Sereni spiega il senso della sua personale ricerca e conclude:

Un'ultima annotazione, riguardo alla scrittura femminile, araba fenice di cui nessuno, fino a oggi, è stato in grado di definire la specificità. Non proverò dunque a farlo io: mi limito a dire di una mia ricerca, cominciata con Casalinghitudine in maniera pressoché inconsapevole, e poi proseguita in maniera più cosciente. Quello che tento di fare è recuperare la "lingua perduta delle donne", una lingua che forse non è mai esistita in quanto tale e che non si può inventare a tavolino, ma che ha trovato infine possibilità di espressione nei linguaggi extraverbali: il cibo, l'abbigliamento ecc. Cercare questa lingua vuol dire scegliere l'ottica delle donne, vuol dire - ad esempio - raccontare più i gesti che le parole, vuol dire provare ad andare al di là di una sovrastruttura culturale inevitabilmente maschile, in cerca di una specificità di genere che mi appartiene, anche se non riesco a riconoscerla fino in fondo, e alla quale appartengo ${ }^{[21]}$.

Nell'insolita confessione che precede Taccuino di un'ultimista (le altre opere non sono introdotte da una così chiara, esaustiva e personale introduzione), la Sereni racconta ancora la difficoltà ma anche la gioia di chi si presta quotidianamente al servizio del prossimo, di chi s'impegna a favore degli "ultimi". "Ultimista", il neologismo coniato per

[17] Per quanto concerne il rapporto con il reale, basti il seguente esempio. Lo ricaviamo da Passami il sale, opera nella quale la Sereni smantella l'ultimo mito positivo della politica: il fatto che, nonostante tutto, si tratti dell'attività umana più concreta e più utile. A volte naturalmente lo è, ma spesso risulta terribilmente astratta, consistendo in interminabili riunioni, strategie sofisticate e incomprensibili, discorsi sempre allusivi, mai trasparenti. Quando, dopo le prime pagine, fitte di riunioni e incontri politici, ci imbattiamo nell'immagine della madre e del figlio che sbucciano patate, abbiamo quasi una folgorazione, ci sembra di assistere a un miracolo di luce. In quel momento, dopo pagine d'irrealtà ingombrante e verbosissima (la vacuità della politica e le "menzogne macroscopiche" dei politici), accade un'epifania quasi miracolosa: quelle patate, così concrete, sono una rivelazione, la prima cosa reale che incontriamo. Molti gesti concreti della quotidianità come questo, che illumina il rapporto tra l'io narrante e il figlio autistico, abitano le pagine della Sereni, dove perfino la nostalgia, così impalpabile, è resa concreta dal ricordo del profumo materno.

[18] Sereni, C., Taccuino di un'ultimista, op. cit., p. 39.

[19] Ibidem.

[20] Ivi, p. 40

[21] Ibidem. 
indicare se stessa e rimarcare la volontà di stare dalla parte degli emarginati di ogni tipo, di quelli che "restano fuori dalla Storia più che mai, benché, probabilmente, più che mai numerosi", potrebbe diventare la definizione comune di tutti coloro per cui l'impegno a favore degli "ultimi" non si riduce a un semplice gesto d'altruismo, ma il cui obiettivo è piuttosto la promozione di microprogetti sociali mirati a risolvere i problemi di persone con difficoltà fisiche e mentali. Lo scopo da perseguire è la costruzione di una possibile società civile. Spiega la Sereni:

Dicendomi ultimista provo a confrontarmi con la loro [degli "ultimi"] espulsione, ad assumere come punto di vista il loro. Stare dalla parte degli ultimi, provare a ragionare con la loro testa la loro pancia e la loro pelle inizialmente mi è capitato per ventura, per un pezzo di me che era ineluttabilmente in gioco più che per cultura o scelta: dichiararmi ultimista significa alla fin fine dirmi che tanto dolore non è inutile, e che a questo mondo finalmente - se non giustizia - può esserci, almeno, una scelta di campo non ambigua ${ }^{[22]}$.

\section{Cibo e memoria in Casalinghitudine: la riappropriazione della storia individuale e familiare attraverso le ricette alimentari}

Le pratiche alimentari costituiscono un'importante risorsa culturale per la costruzione di identità, memoria e appartenenza sociale. Il cibo, che segna imperiosamente con la sua presenza i riti e le stagioni, le continuità e le svolte, è cultura legata alla materialità, al saper fare, all'uso delle mani, alle abilità di mescolare sapientemente ingredienti e saperli dosare in un equilibrio di sapori e colori, di elaborare pratiche culinarie legate alla quotidianità e all'ordinarietà della vita sociale e di relazioni. Attraverso il processo di preparazione del cibo si radica il proprio vissuto alimentare in uno specifico contesto storico, geografico e affettivo. Parlare di cibo significa accostarsi alla memoria del fare, del cucinare, del preparare piatti e ricette che sono stati trasmessi attraverso le generazioni e si sono rielaborati con il tempo, significa recuperare e salvare parole e saperi relegati sovente in spazi di marginalità e in ambiti esclusivamente domestici. Una trasmissione di saperi e tradizioni prevalentemente al femminile, perché le ricette e i piatti sono legati alla costruzione dell'identità femminile, ai rapporti di comunità e familiari, alla dimensione di cura, di saper fare delle donne. Ma il cibo e la sua preparazione hanno altresì una dimensione narrativa, in un costante rapporto tra storia e memoria. Il cibo diventa così memoria, racconto, parole per descriverlo, mentre la sua preparazione è tradizione ma anche innovazione e creatività.

Nel 1998 si è svolto a Soave un convegno incentrato sui Soavi sapori della cultura italiana. Nei contributi gli studiosi hanno confermato quanto il cibo, come regolatore dei rapporti tra le persone (può esprimere un'ampia gamma di rapporti possibili, dal dono alla violenza), la sua preparazione, il ruolo che l'atto del mangiare assume nella vita quotidiana, siano presenti nella letteratura italiana non di rado come temi principali, che consentono agli scrittori di riappropriarsi della quotidianità, e di trasmettere una conoscenza intellettuale ed esistenziale. Il volume degli atti del convegno è stato curato da Bart Van Den Bossche, Michel Bastiaensen e Corinna Salvadori Lonergan, con la collaborazione di Giovanna Domenichini. Particolarmente illuminante è il saggio $\mathrm{Il}$

[22] Ivi, p. 14. 
cibo come narrativa del Novecento: appunti per una tipologia di Bart Van Den Bossche. Lo studioso evidenzia che il soggetto narrante può usare il cibo come stratagemma per comunicare passioni ed emozioni, per conoscere se stesso e l'altro, che è poi quanto fa la Sereni in Casalinghitudine. Altrettanto interessante è il contributo di Marina Spunta, Sapori della narrativa tabucchiana. L'autrice riflette sul rilancio del cibo nella letteratura e nel cinema e nomina l'esempio della Sereni. Le ricette inserite in Casalinghitudine, secondo la Spunta, " [...] ripropongono la cucina come perno della vita familiare e come metafora di libertà individuale"[23].

Il cibo, la sua preparazione, la cucina, considerata non come spazio di reclusione e di restrizione ma come spazio del potere, dove le donne diventano consapevoli di sé e scoprono la propria identità e autonomia attraverso la creatività, sono fondamentali nelle opere della Sereni, diventando quasi il motore simbolico dell'immaginario ${ }^{[24]}$. In Casalinghitudine, in particolare, per raccontare la propria storia personale, ma soprattutto la storia di una famiglia che ha contato nella storia italiana, in cui ebraicità, antifascismo, comunismo e cultura hanno formato una miscela speciale e difficile da vivere, Clara Sereni ha scelto un'impostazione originale: scruta se stessa e i personaggi attraverso il cibo che mangiano e come lo mangiano con la vocazione, che è della migliore letteratura, di salvare dall'oblio persone, fatti, ambienti. Il cibo diventa segno di comunicazione domestica e di interazione familiare ma anche punto di vista politico, un linguaggio parallelo, in grado di raccontare qualcosa in più o qualcosa di diverso da quello che la parola dice.

Con Casalinghitudine la Sereni trasforma un libro di cucina in uno scandaglio nella memoria personale e storica, in un'analisi della vita domestica e familiare e dei rapporti interpersonali. È un'opera difficile da definire, perché l'autrice non si attiene alle regole prescritte dai generi. Con intelligenza tutta femminile, e coerentemente alla sua natura, che la porta sempre a sconfinare, anche in questo caso la Sereni attraversa i confini. L'opera è stata definita un romanzo autobiografico, intimista, di memorie, un romanzo di formazione o un romanzo-diario, privo però di date e di un percorso cronologico lineare ${ }^{[25]}$. La narrazione, basata sull'attaccamento alla materialità della riproduzione domestica, che diventa una possibile modalità di riappropriazione della propria storia individuale e familiare, è scandita dall'alternarsi di ricette culinarie che raccontano una vita ${ }^{[26]}$. Ogni piatto, ogni preparazione, rievoca un frammento di

[23] Spunta, M., "Sapori della narrativa tabucchiana", in Soavi sapori della cultura italiana, Bart Van Den Bossche, Michel Bastiaensen, Corinna Salvadori Lonergan (a cura di), Franco Cesati Editore, Firenze, 1998, p. 458.

[24] A p. 289 del saggio "Casalinghitudini tra identità e storia: la scrittura pluristratificata di Clara Sereni", inserito in Scrittori italiani di origine ebrea ieri e oggi: un approccio generazionale, Raniero Spleeman, Monica Jansen e Silvia Gaiga, (a cura di), Italianistica Ultraiectina 2, Utrecht, 2007, Federico Pellizzi evidenzia che nella narrativa della Sereni "La cucina, la casa, divengono i luoghi dove si combatte la battaglia tra l'entropia e il racconto, la distruzione e la vita".

[25] In Narciso allo specchio. Il romanzo moderno in prima persona (Bulzoni, Roma, 1989) Ulla Musarra Schröder presenta il romanzo in prima persona che non è lo stesso di un romanzo autobiografico, sebbene imiti le strategie dell'autobiografia. A p. 11 la Musarra Schröeder sostiene che sia "nell'autobiografia che nel romanzo in prima persona il soggetto d'enunciazione non è un'istanza narrativa anonima, ma un io concreto e ben definito", che "in maniera più o meno frequente, con l'uso dei pronomi personali della prima ed eventualmente della seconda persona, con verbi al presente, al passato prossimo e al futuro, e con certi pronomi dimostrativi ed avverbi deittici rimanda alla propria situazione enunciativa". L'autrice divide il romanzo in prima persona in tre tipi che definisce classici: il romanzo di memorie, il romanzo-diario e il romanzo epistolare. Se volessimo definire il lavoro della Sereni seguendo questa suddivisione, avremmo non poche difficoltà, perché l'opera sfugge a una rigorosa definizione di genere. È al contempo un'autobiografia, un romanzo di memorie, un romanzo-diario (scandito dall'alternarsi di ricette e non di date). Anche in questo modo la Sereni attesta la sua autonomia, la volontà di sconfinare e sperimentare, di mescolare "gli ingredienti", come in cucina. L'atto dello scrivere per lei è simile al cucinare. Sono entrambe attività molto creative, che s'intrecciano.

[26] Il romanzo in cui ricette e narrativa s'intrecciano, ossia il ricettario autobiografico o narrativo, sta diventando un genere emergente, tanto che María Claudia André in Chicanas and Latin American Women Writers. Exploring the Realm of the Kitchen as a Self-Empowering Site (The Edwin Meller Press, New York, 2001) parla di "narrativa culinaria". Un'altra nota scrittrice, come la Sereni, ha usato questa 
memoria, un incontro, un periodo. Ne risulta un ritmo narrativo frammentario, un procedere per episodi staccati, dove il racconto autobiografico viene interrotto da vere e proprie ricette di cucina. Le ricette, semplici, quasi elementari, sono presentate per tipologia di piatti e associate ciascuna a frammenti di ricordi. Non sono una cornice vuota, bensì ordinano la narrazione in una semantica vissuta. Non sono un pretesto, ma sono esse stesse radicate nel tessuto della memoria personale. Appartengono alla vita quotidiana, ma hanno al contempo una risonanza più profonda, individuale e collettiva. Le ricette sostituiscono le date, creando la giusta scansione per evocare, registrare e ordinare eventi, situazioni e persone, e diventano strumento di ricognizione non solo del vissuto, ma dei rapporti umani in generale. Pertanto hanno valenza sia antropologica (la cucina e il cibo come prisma delle relazioni sociali e private) sia personale, perché sono risorsa per riconquistare il tempo, un modo per reinventare il quotidiano, per ricordare e superare anche vicende traumatiche del passato e del presente, per ricomporre una totalità perduta.

Il passaggio tra cibo e memoria, tra ricette e narrazione degli episodi cui le ricette associano, non è mai meccanico o artefatto. La Sereni usa difatti la prima persona per raccontare la preparazione di ogni piatto, come a registrare un evento che si sta compiendo, di cui è protagonista e testimone. L'io narrante, alla ricerca di radici eidentità, racconta una "vita a mosaico" contrassegnata da una molteplicità di appartenenze e di rappresentazioni del sé, e si fa carico della responsabilità, della scelta, del giudizio, e al tempo stesso partecipa alla riconquista del corpo, del presente, del futuro, della narrabilità delle cose. Distanza e presenza si nutrono reciprocamente. Il molteplice io narrante filtra esperienze personali e altrui, ricerca il proprio passato e il proprio presente all'interno di una memoria e di una conoscenza mobili. Lo spazio privilegiato di quest'operazione è la cucina che diventa un laboratorio ideale, dove si pratica un'attività combinatoria di ricerca e scoperta, dove si può creare, immaginare e ricostruire. Il cucinare stesso è l'unica attività veramente creativa della "casalinghitudine". Ed anche se il diario di cucina ordina la vita per tipi di cibi, su quest'ordine, su questo sistema, s'innesta un susseguirsi di atti creativi, una ricerca di sviluppo e di futuro, tanto che a volte anche le operazioni e i gesti elementari ("taglio grossolanamente la cipolla" o "friggo le melanzane tagliate nel senso della lunghezza", "trito la cipolla e la faccio imbiondire" o "impasto i vari ingredienti nella zuppiera") sono registrati con l'aggiunta di commenti personali e connotativi. In ogni caso, anche questa registrazione di gesti banali e ripetitivi, così frequenti in cucina, ha una sua ragion d'essere, perché costituisce la base, che non è mai rituale bensì ritmica, per ordinare la materia. Ciononostante, siccome la progressione temporale tipica del diario viene sovvertita (la sequenzialità temporale è destrutturata dall'ordine delle ricette e dalla rapsodicità dei ricordi), la tensione tra i tempi commentativi e i tempi narrativi è evidente, per cui risulta facile la distinzione tra un tempo delle ricette e un tempo del ricordo.

Il titolo dell'opera è ancora un neologismo. "Casalinghitudine" è una parola che non esiste, associa a "casalinga", a "solitudine" e "abitudine". Cos'è la "casalinghitudine"?

modalità narrativa: Isabel Allende in Afrodite. A differenza della Sereni, che intercala ricette e narrazione per cui esse sono inserite tra $i$ frammenti narrativi, l'Allende separa nettamente la parte narrativa da quella ricettaria, e inserisce le ricette alla fine del libro, separatamente. Si può dire che la Sereni, non separando le due parti, sia innovativa. Difatti non si attiene alle regole prescritte dai generi bensì le viola, proponendo l'unione di generi e dunque la rottura di genere. Cercando combinazioni nuove e diverse, anche nella scrittura, come in cucina, la Sereni applica la regola del reinventare, unico sconfinamento possibile. 
Per la Sereni la parola indica "[...] un angolino caldo, un privato che abbia un proprio equilibrio e un proprio agio. [...] Un privato non chiuso al mondo, che anzi si costituisce come premessa per essere dentro il mondo, per guardare ad esso dal punto di vista degli "ultimi" che serve, oggi più che mai, per immaginare utopie e cambiarlo" ${ }^{\text {[27] }}$. In uno dei brani di Taccuino di un'ultimista l'autrice approfondisce la spiegazione del concetto. Descrive puntualmente le incombenze femminili che costringono sempre le donne a essere efficienti, a programmare, prevedere, organizzare. Diagnostica inoltre quella specie di "malattia" che le colpisce per quell'ininterrotto dover scegliere tra desiderio di superfluo e urgenze della quotidianità, che le fa sentire in colpa e che tutte "[...] ci portiamo dentro quando laviamo quel pavimento che forse starebbe tanto meglio con appena un pochino di cera, quando appendiamo un quadro coscienti che prima $\mathrm{o}$ poi ci sarà un vetro in più da pulire, quando scegliamo una pianta che poi andrà potata annaffiata concimata, quando prepariamo un cibo accurato per noi stesse o per qualcuno a cui vogliamo bene"[28]. La "casalinghitudine" rappresenta l'eterno tormento della donna, perché i gesti "[...] "inutili" e creativi della quotidianità sono il bambino fantasioso e un po' anarchico che ci portiamo dentro, incerto del suo destino, dei suoi diritti, delle sue prospettive" ${ }^{29]}$. Il suggerimento è di tenere la "casalinghitudine" entro i limiti, proprio come si fa con un bambino, che ha bisogno di limiti definiti e perfino rigidi. Solo così essa diventa "[...] oltreché luogo delle contraddizioni, anche un piccolo punto di equilibrio, una pausa in cui ci si vuol bene, una vittoria precaria (giustamente precaria) del desiderio sulla realtà. Uno spazio per crescere"[30]. Perché, scrive la Sereni nella pagina conclusiva di Casalinghitudine, «[...] è impossibile una vita solo funzionale, senza piccoli gesti di agio, senza un odore di cura, senza una qualche ricchezza" ${ }^{[31]}$. Forte di questa convinzione può dichiarare: "Così le mie radici aeree affondano nei barattoli, nei liquori, nelle piante del terrazzo, nei maglioni e nelle coperte con i quali vorrei irretire il mondo, nel freezer: perché nella mia vita costruita a tessere mal tagliate, nella mia vita a mosaico (come quella di tutti, e più delle donne) la casalinghitudine è anche un angolino caldo", un angolino "da modificare in ogni momento", perché "se fosse fisso sarebbe morire"[32].

Per la Sereni la "casalinghitududine" è anche quel "[...] desiderio nostalgico e creativo di un mondo in cui "ogni cosa ha il suo posto, e ogni posto la sua cosa"'[33]. Era questo il detto caro a zia Ermelinda, uno dei bellissimi personaggi femminili che s'incontrano nelle pagine dell'opera, dove l'autrice intraprende un processo di appropriazione della storia delle famiglie Pontecorvo-Sereni-Silberberg, partendo da una prospettiva peculiarmente femminile. Anche se l'autrice dichiara che molti particolari delle vicende familiari sono contenuti nei libri, nei trattati, nelle memorie, nei saggi e nei carteggi, e molti protagonisti sono stati "consegnati alla Storia spesso già da vivi" ${ }^{[34]}$, ammette al contempo che quel cospicuo materiale documentario per lei è

\footnotetext{
[27] Così Clara Sereni in un'intervista concessa al trimestrale “DM", edito dalla Direzione Nazionale dell'Unione Italiana Lotta alla Distrofia Muscolare. L'articolo è apparso nel numero doppio della rivista, 146/147, settembre/dicembre 2002.

[28] Sereni, C., Taccuino di un'ultimista, op. cit., p. 67.

[29] Ibidem.

[30] Ivi, p. 68

[31] Sereni, C., Casalinghitudine, Biblioteca Universale Rizzoli, Milano, 2007, p. 160.

[32] Ibidem.

[33] Ivi, p. 154

[34] Ibidem.
} 
insufficiente, non le consente di capire fino in fondo. Avverte pertanto la necessità di fare chiarezza, di comprendere le scelte dei genitori e del padre in particolare, occupato tutta la vita a mascherare la propria fragilità disarmata attraverso una compattezza granitica, monumentale, attraverso una continua cancellazione di parti di sé, del proprio passato, attraverso la sostituzione della realtà con l'irrealtà. La scrittura si manifesta dunque come necessità interna, connessa all'urgenza di ricomporre una storia familiare complessa, che riguarda almeno tre generazioni. La scrittura diventa terapeutica. Il passato non è separato e allontanato bensì è cercato, esaminato e ricollocato nel presente. Riportando esistenze e vicende dal passato al presente, l'autrice ricompone il rapporto con le proprie radici attraverso un dialogo che è esistenziale e al contempo letterario.

Zia Ermelinda, detta Mela per "le sue guance, tese e fresche malgrado gli ottant'anni" [35], è stata di certo la figura femminile più importante per la Sereni. Di lei ricorda addirittura l'odore, fatto di colonia e di sapone di Marsiglia. Pianista, ex dama di compagnia della regina, maestra elementare, poliglotta, viveva nell'appartamento attiguo a quello della famiglia dell'autrice. Anche se la memoria familiare tramanda di lei "una immagine di donna dura, avara, dispotica, complessivamente poco simpatica"[36], il ricordo della Sereni è diverso: "Per me zia Mela resta un profumo, la musica, dei gesti eleganti, la sensazione di qualcuno che chiede molto ma molto è disposto a dare, il calore di sentirmi prediletta e unica" ${ }^{\text {[37] }}$. Zia Mela incarna il modello femminile della donna che, libera dalla maternità, può concedersi il lusso di dedicarsi a se stessa senza risparmio, abbinando il piacere per le cose belle e futili all'amore della cultura. Sempre accurata nel suo aspetto è l'unica che, anche dopo un lungo pranzo in famiglia, appare "ancora perfetta nell'abito, nell'acconciatura laboriosa, nel velo di cipria che le satinava le guance"[38]. "Doveva fare di me un genio" scrive la Sereni, "dunque mi rendeva partecipe: della sua toilette, porgendole forcine e fermagli; del suo farsi bella, e ogni tanto mi faceva accarezzare la seta antica dei suoi kimono giovanili; della sua eleganza, fatta di zibellini, volpi argentate, perle matte e broches di rubini [...]"[39].

A zia Ermelinda, senza figli, colta e raffinata moglie di industriale, si affianca la sorella Alfonsa, la forte nonna paterna, figlia dell'alta borghesia ebraica, che nel 1904, in seguito alla morte della piccola Velia, prenderà un lutto mai più dismesso, quasi una premonizione della morte di altri figli. Per questo vestirà sempre di nero, anche dopo aver partorito di nuovo, Enzo nel 1905 ed Emilio nel 1907. Tutto quel nero, per lei, era una difesa, un modo "di cancellarsi, di oscurarsi agli sguardi e delegare ad altri la vita che lei non avrebbe vissuta, la luce piena della ribalta e gli applausi che dovevano venire" ${ }^{[40]}$. I suoi erano "abiti informi a più strati, lunghi quasi fino a terra" ${ }^{[41]}$, ricorda l'autrice. Anche le scarpe erano rigorosamente nere, da uomo, con i lacci. Alfonsa era la "sorella brutta di due sorelle bellissime (zia Elena e zia Ermelinda), e faceva di tutto per apparire più goffa, più sgraziata, più inelegante"[42]. Era una donna severa e poco espansiva che "aveva usato la sua esistenza per essere coraggiosa, per affrontare le scelte dei suoi figli, per accettarne

[38] Sereni, C., Il gioco dei regni, Biblioteca Universale Rizzoli, Milano, 2007, p. 22.

[39] Sereni, C., Casalinghitudine, op. cit., p. 31.

[40] Sereni, C., Il gioco dei regni, op. cit., p. 43.

[41] Sereni, C., Casalinghitudine, op. cit., p. 15.

[42] Ibidem. 
la morte" ${ }^{[43]}$. La Sereni confessa che da bambina quella nonna le faceva paura. Per fortuna la vedeva di rado perché viveva in Israele, dove allevava polli. Imponeva a sé e agli altri una ferrea disciplina, tanto che, ricorda, il "peggior castigo di cui mio padre potesse minacciarmi era: "lo scriverò a mammà"' [44] . Aveva educato i tre figli, Enzo, Enrico ed Emilio, il padre dell'autrice, nel segno della responsabilità, ma anche nella competizione tra loro e il mondo esterno, coadiuvata dalla sorella Ermelinda che, pur essendo nella sua struttura completamente diversa dalla sorella, ne condivideva e appoggiava i metodi sicché "sui ragazzi il suo progetto coincideva in larga parte con quello che Alfonsa aveva in mente" ${ }^{[45]}$. Ermelinda seguiva i tre ragazzi Sereni "con attenzione vigile, in cerca, fra quei nipoti, di qualcuno che potesse diventare, con gli aiuti opportuni, l'Eletto" ${ }^{[46]}$.

Diverse com'erano in apparenza, le univa la volontà comune - quasi un'ossessione - di scegliere per loro il meglio, perché dovevano essere i migliori. I più bravi a scuola, i primi (benché sempre i più piccoli d'età, classi saltate a ripetizione per arrivare più in fretta, e non ci si chiedeva dove), su questo non c'erano difficoltà né dubbi ma non bastava: perciò i libri e la ginnastica, gli abbonamenti ai concerti, quel modo di rendere familiari altre lingue già da bambini, nei giochi e nelle chiacchiere della casa $[\ldots]^{[47]}$.

Della galleria di personaggi femminili fa parte anche Xenia, solo accennata, la nonna materna, che è stata una giovane rivoluzionaria russa, arrestata e deportata dalla polizia dello zar in Siberia, dove ha conosciuto suo marito, Lev Silberberg, social-rivoluzionario ebreo morto durante la rivoluzione del 1905, cui lei stessa aveva partecipato. Dal loro matrimonio era nata Xenia ovvero Xeniuška, la madre della Sereni. Rimasta vedova, Xenia si ritira dall'attività cospirativa per poter offrire alla figlia una vita più tranquilla. Dopo aver soggiornato in diversi paesi dell'Europa orientale e occidentale, si ferma a Roma, dove continua a essere politicamente attiva. Scrive per riviste, prende parte attiva a riunioni e attività di propaganda. La pensioncina che gestisce per vivere al centro di Roma funge da punto di riferimento soprattutto per i profughi ebrei in fuga dalla Russia. Finito con la salita al potere di Stalin il sogno della Nuova Russia, Xenia abbraccerà la causa sionista, senz'essere ebrea. Il sionismo sarà la sua nuova utopia. In età adulta, troverà la sua nuova patria in Palestina, dove contribuirà alla realizzazione dell'utopia egualitaria dei primi kibbutz. Da bambina la Sereni la vedeva raramente, quando insieme a nonna Alfonsa arrivava dalla Palestina, dove viveva. Nonostante ciò, tra tutte le donne che hanno attraversato la sua vita, la Sereni scoprirà nella nonna materna, così poco frequentata, la figura più vera della sua famiglia: una donna che, differentemente da nonna Alfonsa e da sua madre, non si sarebbe mai adattata a essere un'eco, un prolungamento della volontà del marito.

Avere quattro sorelle non rende felici, ci si può sentire addirittura più sole. I rapporti dell'autrice con le quattro sorelle sono stati "complessivamente brutti" e "impasticciati" ${ }^{[48]}$. Inesistente il rapporto con la madre, morta quando lei aveva appena

\footnotetext{
[43] Ibidem.

[44] Ibidem. all'altro".

[46] Sereni, C., Il gioco dei regni, op. cit. p. 57.

[47] Ivi, p. 75 .

[48] Sereni, C., Casalinghitudine, op. cit. p. 75.
}

[45] Sereni C., Il gioco dei regni, op. cit. 51. A proposito della scelta di dare a tutti e tre i figli nomi che iniziavano con la lettera "E", la Sereni a p. 43 scrive: "Enrico Enzo Emilio. I tre figli maschi tutti con l'iniziale "E" come una dinastia, un'unica iniziale per tutti: perché crescessero uniti, per l'orgoglio di casata di Lello, o per la riluttanza di Alfonsa a cambiare le cifre sulla biancheria trasmessa dall'uno 
sei anni. Di lei conserva un ricordo vago, mentre la tradizione familiare la tramanda come una "santa, un'eroina, una martire" ${ }^{[49]}$. Quando morì, per l'autrice "era già morta da tanto", e la sua infanzia "[...] era avviata su binari che non prevedevano la sua presenza"[50]. La ritroverà nelle pagine di un suo libro, I giorni della nostra vita, scritto $\mathrm{e}$ pubblicato poco prima di morire con il nome di battaglia di Marina, "per essere vangelo di una generazione di donne comuniste" ${ }^{[51]}$. Agli occhi della figlia, quell'opera appare troppo inquinata da menzogne, censure, omissioni. Un'autobiografia in cui la vita di Xeniuška non esiste, né esiste la nonna social-rivoluzionaria, né il passato sionista. È un'autobiografia incompleta, che comincia solo quando la madre dell'autrice diventa la moglie di Emilio Sereni, conosciuto durante una serata organizzata dalla nonna Xenia Silberberg nella sua pensione per raccogliere fondi tra i ricchi ebrei romani per la costruzione dei primi kibbutz in Palestina, cui il giovane liceale prende parte insieme al fratello Enzo. La vita della giovane Xeniuška prima dell'incontro con Emilio non esiste, nell'opera viene cancellata con un colpo di spugna. C'è solo la vita di Marina, nome di battaglia che Xeniuška ha scelto per sé quando ha deciso di assecondare il marito nelle sue lotte, e con il quale i membri del Partito la conosceranno nella clandestinità.

Non meno importanti nella vita dell'autrice sono stati i personaggi maschili: il nonno paterno, Samuele, detto Lello, ebreo e medico di fiducia del re, che in virtù delle sue "proporzioni minute [...] se lo teneva volentieri accanto nelle foto ufficiali, per dar risalto ai propri centimetri di vantaggio" ${ }^{[52]}$. La tradizione familiare lo tramanda come una figura scialba, riconoscendogli come unico merito quello di "avere formato una generazione intera di madri ai più avanzati criteri di puericultura"[53]. La figura maschile che sta al centro dell'opera è quella paterna. La Sereni cerca di ricostruire il rapporto col genitore per capire le sue scelte di militanza intellettuale e politica. Si sforza di comprendere perché ogni suo gesto di libertà, di autonomia e di intellettualità si scontrava con il disappunto del padre, con il suo furore. Da quel padre, per il quale la parola chiave era il Partito, sempre trincerato nel suo studio dove di guardia, al di sopra della porta, c'era un enorme ritratto di Stalin, non ha mai avuto consensi. Nel tentativo di giustificarlo, la Sereni osserva che forse lui "avrebbe voluto soltanto che percorressi strade diverse dalle sue, ma evitare di sconfinare nei suoi territori non era facile: aveva fatto e sapeva tante di quelle cose" ${ }^{\text {[54] }}$.

La crema di piselli, tra le ricette, è associata a un episodio significativo: un pranzo al ristorante in compagnia del padre, un'esperienza che per la Sereni è stata una rivelazione. In quell'occasione suo padre le sembrò diverso dal solito "per l'agio con il quale si muoveva nel ristorante d'alta classe, per l'alterigia che mostrò nella scelta del vino [...], per la naturalezza con cui pagò il conto" ${ }^{[55]}$. Era un'altra persona, diversa dall'uomo tutto d'un pezzo che si sforzava di apparire e che lei conosceva. Capì allora che era possibile avere anche una doppia vita, che anche suo padre aveva una personalità franta. Se da una parte era "il grande, scintillante parlatore di cui mi si cominciava a favoleggiare,

[49] Ivi, p. 49

[50] Ibidem.

[51] Sereni, C., Il gioco dei regni, op. cit., p. 419.

[52] Sereni, C., Casalinghitudine, op. cit., p. 37.

[53] Ibidem.

[54] Ivi, p. 72

[55] Ivi, p. 41. 
il Maestro capace di trasfondere cultura a generazioni di allievi", dall'altra era un uomo di mondo, un "buongustaio esigente", anche se poi "a casa imponeva diete monotone e cibi insipidi”[56]. Per l'autrice quel pranzo fu una folgorazione. In quell'insolita versione, il padre svela la propensione per i piccoli piaceri della vita e per un sobrio edonismo, fino ad allora soffocati da un'irreprensibile severità di comportamento. Alla figlia appare finalmente umano, più vero. Inonda la zuppa di piselli con le lacrime e in sua presenza, per la prima volta, si concede un atto trasgressivo: si permette di fumare una sigaretta.

L'opera si chiude con la morte del padre e la descrizione minuta della cerimonia funebre, che si svolge in un giorno in cui la primavera pare esplodere su Roma.

Alle tre del pomeriggio, durante l'orazione funebre, il sole picchiava forte sul mio loden grigio, sugli stivali improvvisamente stretti: avevo gli occhi annebbiati dal polline, temevo il brusco insorgere del raffreddore da fieno [...] Osservavo gli effetti cromatici del compromesso storico, niente bandiera rossa sulla bara, niente fiori rossi nei suoi pressi: tricolori, nuances dal rosa al giallo al violetto [...] Tornammo dal cimitero in taxi, le strade con i manifesti listati a lutto, sul sedile del conducente il giornale piegato sul necrologio. La risonanza pubblica attutisce i tremiti interni ${ }^{[57]}$.

Nella parte conclusiva di Casalinghitudine, intitolata non a caso Conservare, la Sereni denuda ancora la sua anima, soffermandosi a descrivere il rapporto difficile con quel padre ormai uscito dalla sua vita, esigente e "scomodo", che le imponeva di "agire con la sua stessa scientificità, quella che applicava al lavoro politico, al lavoro culturale, forse anche agli affetti" ${ }^{[58]}$, mentre lei pretendeva di essere forte, autonoma, intellettuale. Comunque libera e diversa. Una figura forse fin troppo ingombrante, quel padre, che "esorcizzava con i però i sentimenti, schiacciandoli sotto il Partito o la Storia, magari la Storia dell'alimentazione"[59]. Una figura con la quale il confronto è stato sempre difficile, tanto che la Sereni confessa d'aver impiegato un certo numero di anni per non essere "la figlia di" e per ritagliarsi un minimo di autonomia. Ciononostante, quel padre è una figura che si deve "conservare" intatta nella memoria, come i profumi, i colori, le manie, le piccole fonti di calore, che la Sereni ritiene indispensabili al pari delle grandi.

Cala il sipario. Alla fine, la Sereni si chiede se suo padre abbia creduto davvero e fino in fondo alle scelte intransigenti compiute nella vita, quelle che hanno condizionato anche l'esistenza dell'autrice e della famiglia. Resta il dubbio: "Chissà: forse anche mio padre al Sol dell'Avvenire alla fine ci credeva meno, o in modo diverso, certo molte maglie della sua rete si erano strappate, io fra le tante. Le mie improvvisazioni la mia fatica a vivere e la sua scientificità, il suo suicidarsi di silenzio, una guerra senza quartiere fino all'ultimo. Lui non ha vinto; io, mi limito a vivere"[60].

\section{Storia, memoria, costruzione dell'identità ne /l gioco dei regni}

L'operazione di recupero e ricostruzione della memoria familiare, già avviata in modo originale in Casalinghitudine, prosegue nel romanzo Il gioco dei regni, uscito per la prima volta nel 1993. Nella Prefazione, inserita nella ristampa del 2007, Alberto Asor

[56] Ibidem.

[57] Ivi, pp. 142-143.

[58] Ivi, p. 149.

[59] Ivi, p. 89.

[60] Ivi, p. 157. 
Rosa definisce l'opera "uno dei più bei libri italiani degli ultimi vent'anni, e il più bel libro di memoria famigliare ebraica accanto a Lessico famigliare di Natalia Ginzburg" ${ }^{[61]}$. Tecnicamente parlando, Il gioco dei regni è un romanzo genealogico, una saga di famiglia basata sul montaggio sapientemente orientato e perfettamente riuscito di brani tratti da un cospicuo e originale materiale documentario di cui fanno parte lettere, diari, memorie, testimonianze, documenti ritrovati in archivi pubblici, documenti infantili dei vari protagonisti e persino pagine del libro pubblicato dalla madre dell'autrice, I giorni della nostra vita, qui già citato. Questi molteplici materiali confluiscono tutti nel libro, cuciti insieme da un tessuto narrativo essenziale, nel quale prevale la continua e ansiosa interrogazione dell'autrice che pratica una scrittura liscia, ordinata, fatta di parole talvolta taglienti perché dolenti e impietosamente scarne, una scrittura che abbatte qualsiasi distanza tra chi scrive e chi legge creando momenti di grande coinvolgimento e tensione emotiva. Una scrittura che non sublima la fatica del riscatto, ma di questa anzi si sostanzia.

Nel raccontare la storia avvincente e romanzata di una dinastia, risalendo indietro di tre generazioni, la Sereni è impegnata a spiegare, prima a se stessa che ad altri, la responsabilità di sentirsi l'erede di una famiglia emblematica e fuori dall'ordinario, straordinariamente legata alla storia del Novecento di cui ha segnato le svolte principali. Lo scopo è testimoniare la difficoltà e la sofferenza, ma anche l'orgoglio, dell'"essere una Sereni". La molteplicità dei contributi, come osserva Asor Rosa, "non inficia l'unità narrativa dell'insieme, che è straordinaria", ma serve piuttosto a "dare spazio onestamente anche alla pluralità delle voci e degli accenti" ${ }^{[62]}$. In quest'opera mista, ibrida, contaminata, nella quale la Sereni si muove su due piani paralleli, quello della documentazione e quello dell'invenzione, essa supera ancora una volta i rigidi steccati dei generi, e mette a dura prova la sua inesausta carica affabulatoria. Da questa nuova esperienza l'autrice esce ancora una volta vincente, in virtù del ricorso a una lingua fortemente comunicativa, a un lessico molto tradizionale, che "[...] evita di proposito asprezze e dissonanze, per una narrazione incandescente che già in sé ne contiene troppe"[63], e in virtù della scelta di affidare il racconto a una prosa calibrata, "ben educata e rotonda"[64].

Il gioco dei regni è un libro fondato principalmente sul ritrovamento della memoria perché, come recita il testo tratto dalla tradizione orale dei Hassidim che funge da epigrafe all'opera, "tutto quello che so fare, è tener viva la memoria di questa storia" ${ }^{[65]}$. Uno dei temi profondi dell'opera è dunque la memoria individuale che si fa al contempo memoria collettiva. Una vita senza memoria è inevitabilmente una vita senza qualità, nella quale l'oblio e l'alienazione inchiodano l'individuo a una percezione incompleta del presente. Salvaguardare il passato, fare chiarezza su quello che è stato, è anche un modo per costruirsi una trama razionale su cui prospettare l'avvenire. La memoria rappresenta un bene insostituibile e la sua narrazione, come ricorda Claudio Magris, è una sorta di guerriglia contro l'oblio. Narrare la memoria significa conservare il ricordo, lottare contro la morte, vuol dire operare una scelta, ossia scegliere della vita, propria e altrui, quello che merita di essere conservato e reiterato, fatto continuare per essere trasmesso

\footnotetext{
[61] Asor Rosa, A., Prefazione, in Il gioco dei regni, op. cit., p. III.

[62] Ivi, p. IV.

[63] La Porta, F., La nuova narrativa italiana. Travestimenti e stili di fine secolo, Bollati Boringhieri, Torino, 1999, p. 129.

[64] Ibidem.

[65] Dal testo posto in apertura de Il gioco dei regni, tratto dalla tradizione orale dei Hassidim.
} 
ai posteri.

Questa necessità di raccontare per comprendere e collocare nella storia la sente anche la Sereni, che nell'ultimo capitolo de Il gioco dei regni, in una esaustiva postfazione, spiega le circostanze e le ragioni che l'hanno indotta a intraprendere l'avventuroso viaggio alla ricerca della memoria perduta, indispensabile alla costruzione di un sé più completo e consapevole. Un'esperienza non facile, a tratti dolorosa, perché gli snodi del percorso producono, come confessa, "ogni volta una scossa, ogni volta la necessità di rimettere a posto i pezzetti dentro di me: perché tutto mi è stato raccontato in maniera diversa dal reale, e per ragioni non sempre comprensibili" ${ }^{[66]}$. Per questo decide di risalire indietro di tre generazioni, per conoscere meglio la storia dei suoi parenti, del padre e della madre in particolare, dei quali ha un'immagine troppo solenne e accreditata, quella trasmessa dalle ricostruzioni stereotipate dei libri di storia verso i quali la Sereni mostra diffidenza, perché selezionano, escludono, incapsulano le persone in ruoli, in personaggi a tutto tondo, senza ombre o incertezze. Per questo, informa l'autrice, “[...] avevo chiesto di incontrare Giorgio Amendola per sapere di loro, di mio padre e di mia madre com'erano stati davvero e non soltanto come si erano consegnati alla Storia"[67]. Recupera materiali storici e fonti ufficiali d'archivio, lettere, interviste, documenti, e li mescola ai ricordi d'infanzia, cerca di immaginare, inventa situazioni e dialoghi, affidandosi a quello strumento che lo storico di professione deve ripudiare, ma di cui il romanziere non può fare a meno: l'immaginazione. Già in Casalinghitudine l'autrice aveva confessato che dei personaggi canonizzati non sapeva che farsene, rivendicando un ruolo insostituibile, per capire davvero la storia, all'immaginazione: "Nella memoria mia madre è sempre stata morta; mio padre è sempre stato, se non vecchio, comunque a rischio della vita, affaticato, occupato. Il prima, quel nucleo nascosto che pure esiste da qualche parte dentro di me, posso solo immaginarmelo, raccontarmelo come una fiaba"[68]. Pertanto, il ricorso all'immaginazione, lungi dall'essere un semplice espediente, assume una funzione cognitiva o addirittura ermeneutica: consente di ricostruire la storia vera, che abbia una sua coerenza, faccia ordine dove la vita e le passioni hanno generato disordine, censura, oblio, consente forse di capire. $\mathrm{O}$ almeno di tenere vivo il ricordo. Ripercorrere, ritrovare, reinventare la storia di due famiglie, quella del padre e quella della madre, sullo sfondo della prima metà del Novecento significa, per la Sereni, cercare le radici del proprio modo particolare di essere donna, dare senso alla scelta di essere ebrea, collocare la propria vocazione all'utopia in una continuità che dia ad essa forza $^{[69]}$.

Protagonisti di questa saga familiare sono i tre fratelli Sereni, nati agli inizi del Novecento, Enrico, Enzo ed Emilio, il loro padre Samuele, clinico famoso e medico della Real casa, la loro madre Alfonsa Pontecorvo, i loro figli e poi via via i parenti vicini e lontani, gli amici d'infanzia e di maturità. Parallelamente si snoda la storia della famiglia

[66] Sereni, C., Il gioco dei regni, op. cit., p. 421. Il corsivo, qui come altrove nelle citazioni, è nel testo. Si noti, nel brano citato, come la memoria non sia affatto data per scontata. Essa ha i suoi giochi, talvolta innocui, talaltra ingannevoli: può tramandare la vita che poteva essere e non è stata, la vita che ancora si spera per sé e per gli altri, può ingigantire o ridurre le sensazioni provate, la percezione di un evento. Ma a condizione di farla interagire con altre fonti, come la Sereni fa in questo libro, la memoria ha sempre pieno diritto di parola sul piano della conoscenza dei fatti.

[67] Ivi, p. 418.

[68] Sereni, C., Casalinghitudine, op. cit., p. 50.

[69] Resta quasi del tutto fuori dall'opera l'esperienza del rapporto con l'handicap, anche se le pagine dedicate al fratello del nonno paterno, Settimio, malato di mente, esprimono la volontà di non tacere del tutto una realtà non estranea alla storia della famiglia. Settimio, sin dalle prime pagine del libro, è descritto come "un bambino intrappolato in un corpo adulto" (p. 23). 
Silberberg, la famiglia della madre della Sereni. Le storie delle due famiglie si fondono a metà libro, quando Emilio, il più piccolo dei fratelli, detto Mimmo o anche biblicamente Uriel, napoletanizzato in un affettuoso Uriello, sposa Xenia (Xeniuška) Silberberg, che si convertirà all'ebraismo per poterlo sposare in sinagoga, che da combattente clandestina diventerà Marina, mentre nell'ambito privato sarà Loletta ${ }^{[70]}$. La Sereni pone in particolare evidenza l'indissolubilità di questa coppia, che è fondata su un patto che li vede protagonisti esclusivi, fra loro totalmente solidali e complici: "Mimmo e Xenia resistono ad ogni urto, e il loro stare insieme agli occhi di chi li frequenta assurge a simbolo quasi magico della coppia "nuova", capace di assorbire al proprio interno ogni trauma e ogni amarezza, guadagnandone persino in ricchezza e solidità" ${ }^{[71]}$. Un rapporto che esclude tutti, il loro, anche le tre figlie: "Dentro, Uriello e Loletta, soli. Tutti gli altri fuori, gli amici fraterni e i compagni e le figlie e Xenia [la madre di lei]..." ${ }^{[72]}$. Sullo sfondo di questa reciproca e assoluta solidarietà, la comune dedizione altrettanto assoluta al Partito comunista, ovvero, per antonomasia, il Partito, "simulacro vivente di liberazione futura $[\ldots]$ cui si riconosce ogni diritto anche quando sottopone a dilazione, prigionia, tortura" ${ }^{\text {"[73] }}$. Entrambi si sottomettono alle imperscrutabili decisioni dei vertici del Partito che non si discutono, accettano ciecamente la dialettica comunista, capace di giustificare tutto, Emilio dopo esser passato attraverso la raffinata educazione talmudica ${ }^{[7]}$. Quando si tratterà di avviare un processo di rinnovamento e riforma, da personaggi come Emilio il Partito prenderà velocemente le distanze. Sereni sarà vittima di questo cambiamento di rotta: chiuso in una rete di doveri e rigidezze, si ritirerà amareggiato e sconfitto. Nei brani che di seguito riportiamo, tratti dal trentasettesimo capitolo della sezione intitolata Ballata a Loletta, la Sereni descrive con partecipazione emotiva il dolore del padre, che vede crollare di colpo tutti gli ideali in cui aveva fortemente creduto, che avevano condizionato le sue scelte, alcune molto dolorose, finanche di rinuncia degli affetti più cari.

Attorno a lui il mondo intero, inesorabilmente, cambiava: per inciampi, per evoluzione, per catastrofi. Non ammise mai di aver smesso di credere: non nel '56, quando l'Ungheria fu invasa e l'obbedienza significò allontanamenti e censure; non nel '67, quando la guerra in Medio Oriente gli deflagrò dentro, e scelse le ragioni del Partito negandosi a quelle degli affetti; non nel '68, quando anche in casa le passioni del comunismo si delinearono diverse, e intanto i carri armati occupavano Praga.

[...] Non lo assolveva il Partito, che per vestire nuovi panni in fretta e senza sottilizzare doveva disfarsi dei vecchi. Non lo assolveva la famiglia - quella di qui come quella di lì - che aveva pagato care, forse ad usura, le sue scelte.

Quando gli strappi alla sua rete, sommandosi, gli resero irriconoscibile il mondo, si separò da tutti i suoi libri, se ne allontanò; e fu come farsi cieco.

[...] Attorno a lui metri e metri e metri di librerie svuotate, deserte; sui muri, i segni di quello che non c'era

[70] Al momento della sua scomparsa, a commento dei tre nomi che la madre usava, a p. 67 di Casalinghitudine la Sereni annota: "Lasciava dietro di sé un nome difficile (non una delle mie pagelle ne reca la grafia esatta); una fama di donna coraggiosa, innamorata di cose belle che mai, nella sua vita avventurosa, aveva avuto; di donna piena di gusto e sensibilità, capace come Rossella O'Hara di inventare un vestito da una tenda. E di pessima cuoca".

[71] Sereni, C., Il gioco dei regni, op. cit. p. 323.

[72] Ivi, p. 389.

[73] Asor Rosa, A., Prefazione, op. cit., p. VII.

[74] Già in Casalinghitudine, per spiegare l'adesione incondizionata del padre alle regole del Partito, a p. 52 la Sereni scrive: "Per sé aveva accettato tutto, le torture e la morte e le contraddizioni insanabili, a tutto era riuscito a dare un senso, come se ogni avvenimento, ogni pensiero fosse la maglia di una rete che abbracciava il mondo". 
più. Polvere, sporcizia, e i residui di una vita: la musica, il ritratto di Xenia, i giornalini dei Regni.

[...] Ordine e disciplina comandamenti vuoti, via via più staccati da un progetto. Un dolore che dilagava, una solitudine feroce.

Fino all'ultimo ${ }^{[75]}$.

Seguendo la linea materna, nella quale si riconosce meglio ("Riflettevo sulla genealogia femminile, da mia nonna a mia madre a me" ${ }^{\text {"76] }}$ ), ma senza escludere la linea paterna, la Sereni ricostruisce dunque gli episodi e i caratteri, ricrea le emozioni e descrive i fatti, per approdare a una verità, la sua verità, che è diversa da quella ufficiale, tributaria di censure, pudori, ideologie, opportunità del momento. La storia vissuta da tre generazioni di Sereni spazia così dalla rivoluzione russa, alla quale la nonna materna ha partecipato attivamente, alle lotte antifasciste, in cui sono stati coinvolti entrambi i genitori, militanti comunisti, fino alla persecuzione razziale di cui è stata vittima la famiglia paterna. Una quantità di storia imponente, che potrebbe schiacciare le protagoniste principali, la nonna Xenia e la madre dell'autrice, alla cui personalità la Sereni vuole invece conferire spessore umano e psicologico. Una storia imponente che, lungi dal renderle eroine, ha fatto di queste donne le eroiche vittime delle inevitabili ripercussioni dei grandi avvenimenti sulle vite private.

Scavando nell'oscurità del non-detto, del taciuto, del rinnegato, la Sereni cerca le tracce materiali dei suoi familiari con la pazienza di un archeologo e la curiosità di un esploratore. Le vere eroine di questa delicata operazione, di questo libro di "finzione", però molto più vero di molti testi di storia, sono le donne. Nel romanzo la Sereni disegna un'appassionata immagine di militanza femminile. Non è solo la madre Xeniuška (alias Xenia da adulta, o ancora Marina da combattente e clandestina o Loletta, soltanto "nome d'amore"[77], usato nell'ambito degli affetti domestici) a incarnare la figura della perfetta militante. Figure di militanti altrettanto convinte sono le due nonne, quella paterna, Alfonsa, e quella materna, l'altra Xenia, una figura da reinventare per raccontare "[...] com'era stata - una rivoluzionaria - e non come la piccola donna noiosamente iperprotettiva che mia madre aveva descritto nel suo libro" ${ }^{\text {[78] }}$. Tutte e tre praticano con ossessiva devozione un'unica virtù, che divora e annulla il resto, e divora le loro stesse vite: la virtù della coerenza. Sono rappresentate senza contrasti: militanti della maternità (Alfonsa, la nonna paterna), dell'amore (Xenia, la nonna materna), della politica e del Partito (Xeniuška, alias Marina, la madre della Sereni). Le tre donne incarnano l'assoluta fedeltà a una causa, e un certo tipo d'inflessibilità femminile che dal focolare domestico si trasmette al focolare ideologico. Mai in loro il minimo dubbio, il pentimento non è contemplato. Alfonsa incarna la madre perfetta. Niente e nessuno può relativizzare il suo

[75] Sereni, C., Il gioco dei regni, op. cit., pp. 406-407.

[76] Ivi, p. 420.

[77] Sereni, C., Casalinghitudine, op. cit., p. 50

[78] Sereni, C., Il gioco dei regni, op. cit., p.419. L'opera della madre cui la Sereni allude è Il libro della nostra vita, La Nuova Italia Editrice, Firenze, 1970, già pubblicato nel 1955, Edizione di Cultura Sociale. Ne Il gioco dei regni la Sereni ricostruisce la vita di Xenia, la nonna russa materna, della quale non ha alcun ricordo, sulla scorta di documenti reperiti durante un viaggio in Israele dove, in un archivio, trova custoditi ventotto scatoloni con materiali inerenti la sua vita e la sua attività. Nel piccolo cimitero del kibbutz riservato agli ebrei, visita la sua tomba. Sulla lapide, il solo il nome è scritto a grandi lettere, come di chi sia talmente amato e conosciuto da non aver bisogno di cognome. Ne Il libro della nostra vita, la figlia Marina descrive Xenia non come era stata veramente, una donna forte e coraggiosa, capace di grande abnegazione e amore verso il prossimo, ma solo come "[...] una madre qualsiasi, uggiosamente protettiva, della quale liberarsi al più presto per essere finalmente adulti. Come se il rapporto con la madre non avesse peso, come se i legami che le univano fossero di sangue soltanto, e incidentali”, spiega la Sereni a p. 179 de Il gioco dei regni. La Sereni non ha mai perdonato la madre per averle negato l'opportunità di conoscere questa nonna, per la quale prova affetto e stima. La storia di Xenia Silberberg, morta in Palestina nel 1957, è stata raccontata da Yakov Viterbo in un libretto intitolato Storia di un'ebrea non ebrea. 
rapporto con i figli, che ha abituato alla sua eterna presenza e si aspettano pertanto "che il suo latte, come quello della centenaria Sara moglie di Abramo, per miracolo scorra a fiumi finché ne avranno bisogno, finché il suo nutrimento non li sazierà e farà grandi, ancora più grandi..." [79]. L'unica cosa di cui Alfonsa era veramente fiera e "acutamente consapevole era il suo ventre" ${ }^{[80]}$ scrive l'autrice, quella "zona calda" dove crescevano i figli, i suoi unici tesori.

Xenia, la nonna russa, incarna invece l'icona della donna che ama incondizionatamente, prima il suo compagno Lev, dolce e rivoluzionario, poi la figlia aspra e rivoluzionaria, che la respinge e la rinnega crudelmente in una lettera terribile, con cui tronca ogni rapporto con lei, rifugiata in Palestina, sebbene, come abbiamo già detto, non ebrea: "Ho il diritto io, in quanto membro del Partito, di mantenere rapporti con le persone che possono danneggiare seriamente la mia azione, danneggiare seriamente l'Unione Sovietica?"[81], le scrive Marina nella lettera datata 28 febbraio 1937 in cui la dedizione cieca al Partito, che non si discute, la costringe a rinunciare agli affetti più profondi. Scrive ancora Marina alla madre nella stessa missiva: "Quando dico che metto il Partito al di sopra di te, non ho dubbi" ${ }^{[82]}$. La figlia non può permettersi di "mantenere rapporti con persone che possono danneggiare seriamente l'Unione Sovietica”. Xenia, la madre, che ha consumato un'esistenza inseguendo il miraggio della rivoluzione, nell'URSS di Stalin è diventata controrivoluzionaria. Con il nemico, chiunque esso sia, il Partito non può permettersi alcun contatto. Non resta che obbedire e ammutolire. Nonostante il ripudio della figlia, che la sacrifica al volere mistico del Partito, la militanza dell'amore e del dare di Xenia non s'arresterà, sarà interrotta solo dalla morte della figlia, che morirà di cancro. Non una morte eroica, da combattente rivoluzionaria, bensì "stupida, banale" ${ }^{[83]}$, commenta la Sereni. Volontariamente e sistematicamente esclusa dal patrimonio di ricordi che viene consegnato a Clara, in seguito all'ostracismo decretatole a causa della sua scelta sionista, sarà la scrittrice a riscoprire la ricca personalità della nonna Xenia nel viaggio in Isreale, di cui racconta nell'ultimo capitolo, e a riportarla letteralmente in vita attraverso una ricerca appassionata di documenti e testimonianze, cui si affianca lo sforzo di immaginarne le sofferenze, le frustrazioni, le speranze, il coraggio.

Anche prima di questa scelta di rinuncia estrema da parte di Marina, il rapporto tra madre e figlia era incrinato. Difatti, la madre della Sereni, Xeniuška alias Marina, si ritiene vittima di un girovagare di cui non capiva le ragioni, di un vivere randagio che le ha impedito di costruirsi un sentimento di appartenenza (si dichiara addirittura apolide, senza radici né di posizione né di affetti). Non è per niente fiera della madre, che si è ostinata a tener vivo il ricordo del marito, rivoluzionario di professione nella Russia zarista dei primi del Novecento. Per lei, invece, quel ricordo si associa solo a sconfitte e sofferenze, povertà ed esilio: un ricordo racchiuso simbolicamente in uno spelacchiato orsacchiotto di peluche, unico dono lasciatole da un padre mai conosciuto, che l'aveva abbandonata già prima di nascere, condannandola ad essere un'orfana. Nel suo destino, in virtù della vita che aveva scelto, la morte era già prevista. Un figlio era soltanto un

[79] Sereni, C., Il gioco dei regni, op. cit., p. 256.

[80] Ivi, p. 11.

[81] Ivi, pp. 298-299.

[82] Ivi, p. 299.

[83] Sereni, C., Casalinghitudine, op. cit., p. 52. 
incidente di percorso.

L'intelligenza e la cultura, il fascino e l'eleganza a Xenia - così pensava sua figlia - servivano soltanto per soffrire, per penare la vita e farla penare a lei...

Sul cuscino intriso di lacrime, contro sua madre e tutto il mondo che le stava intorno Xeniuška tentò di schierarsi su un altro fronte: risoluta ad essere tutto il contrario di lei, della "compagna Irina" come insisteva a farsi chiamare, stabilì che mai si sarebbe occupata di politica. E per guadagnarsi il principe azzurro, di cui sentiva l'assoluta necessità, decise di sé che sarebbe stata bella, gentile, disponibile, e stupida ${ }^{[84]}$.

Neppure l'incontro con Emilio Sereni, che la soggioga e l'affascina con la sua forte personalità e cambia completamente il suo destino, coinvolgendola in una scelta che richiede assoluta abnegazione e disponibilità ad affrontare tutti i rischi, modificherà il rapporto di Xeniuška con la madre. Anzi, com'è stato rilevato, negli anni duri della guerra fredda, la sua fede nel Partito e le sue regole la porterà a interrompere del tutto i contatti con lei, che ha scelto di vivere in Palestina. Né il conflitto appare superato negli ultimi mesi di vita, quando Xenia è chiamata al capezzale della figlia morente che, pur stremata dalla malattia, non rinuncia a ribadire la sua distanza da lei, la diversità di cui è orgogliosa e fiera. Sono pagine a tratti impietose, da cui sembra trasparire il rimpianto della scrittrice e l'astio verso chi, negandole la memoria, le ha tolto un pezzo delle sue radici. Sono pagine amare, più di quelle cui già in Casalinghitudine la Sereni ha consegnato il ricordo della madre.

La storia, così raccontata, suggerisce ancora una volta l'idea vichiana di ciclicità e ripetitività, non tanto nei fatti, rivoluzioni e lotte che pretendono di cambiare il mondo, quanto nei destini femminili che si perpetuano. Ai grandi avvenimenti politici le donne partecipano, ma insieme ai loro uomini, senza neppure immaginare di poter scegliere tra famiglia e politica, quando i mariti vi sacrificano libertà e vita. Le esperienze della nonna russa e della madre della Sereni sono in questo senso illuminanti: la partecipazione delle donne alla storia resta legata alla dimensione privata, alle scelte del proprio compagno. E se un dubbio le attraversa, viene subito riassorbito, come nel caso della madre della Sereni, dal piacere di essere anche moglie e madre, oltre che militante comunista: "a Xenia vengono alla mente altre donne, quelle che hanno scelto di non smettere la politica in prima persona. Sui prezzi da pagare, nell'un caso e nell'altro, accuratamente Xenia evita di interrogarsi. Le piace tornare ad essere Loletta e nient'altro, amore e pace e rifugio" ${ }^{[85]}$. La Sereni è consapevole di questa sfasatura, che è propria del vissuto femminile nei secoli.

Col lavoro sul recupero della memoria, al contempo familiare e collettiva, la Sereni ha ricostruito un privato che vuole essere anche pubblico, ha messo sotto i riflettori donne che hanno dovuto accontentarsi di essere in secondo piano nelle lotte e nelle scelte di mariti guerrieri, da sostenere. L'autrice ricompone così la scissione tra esterno e interno, casa e mondo. Un'operazione che le consente di rivalutare figure femminili nelle quali meglio s'identifica, e di operare quella riconciliazione tra i due fratelli Sereni che la Storia aveva reso impossibile ${ }^{[86]}$. Solo in un breve capitolo l'autrice riflette sulla [84] Sereni, C., Il gioco dei regni, op. cit., pp. 112-113.

[85] Ivi, p. 323.

[86] Enzo e Emilio Sereni aderirono al sionismo, una scelta divenuta per entrambi totalizzante. Enzo non rinuncerà mai alla sua laicità, pur non portando fino in fondo il progetto che lo condurrà con la moglie Ada in Palestina, dove sarà uno dei protagonisti dell'esperienza dei kibbutz. Divenuto, dopo l'avvento del nazismo, l'organizzatore infaticabile di iniziative finalizzate a salvare dall'annientamento gli ebrei 
frattura insanabile creatasi fra i due fratelli: "Sionismo, comunismo. Parole che hanno assunto negli anni significati e sfumature diversi [...] I vent'anni di Enzo e di Mimmo, i febbrili vent'anni di chi era nato con il secolo che ora sta finendo, trovavano in quelle $\mathrm{e}$ in altre parole un denominatore comune: la speranza di un mondo diverso, più giusto ed umano"[87]. Le scelte dei fratelli di dedicarsi l'uno alla causa comunista l'altro a quella sionista sono meno lontane l'una dall'altra di quanto non possa sembrare. "Chi non fa, non sbaglia": questa è la risposta che la Sereni dà a chi solo critica gli effetti prodotti dalle scelte di allora. Demistificare sì, ma per capire e non per sparare a zero. Eliminare ogni retorica, ma per ridare stima a chi negli "anni spietati" non si è tirato indietro, lasciando un grande esempio di impegno civile accompagnato nella Sereni dalla riflessione sulla laicità e la messa in guardia da ogni dogmatismo.

Lotte feroci dilaniavano i due gruppi, e non soltanto sul piano verbale: fra sionisti, il rapporto con le popolazioni arabe era già una discriminante; fra i comunisti, la cultura del silenzio e del sospetto già mieteva le prime vittime.

Ma per chi cercava un sogno da vivere, per chi voleva essere parte attiva della forza che porta avanti il mondo, una scelta si imponeva comunque: fra potenti e umiliati, fra vittime e carnefici, fra oppressi e oppressori. Quale che fosse il nome che si dava agli oppressi, quale che fosse l'ambito in cui si identificavano gli oppressori.

In ogni scelta, entrarono per ciascuno inclinazioni e sentimenti, esperienze patite e nodi psicologici irrisolti, passioni e timori: ma di questo ben poco è dato sapere, perché chi allora scelse di agire usò le parole per cambiare il mondo, e non per raccontare di sé.

Gli anni erano spietati, il nemico appariva invincibile: nell'affrontarlo a viso aperto, nel decidere della propria vita tutta intera ci fu chi seppe mantenersi laico, e chi mutuò dalla religione il senso dell'unità indissolubile, e del dogma ${ }^{[88]}$.

Il romanzo si conclude con un'immagine di speranza: quell'orsacchiotto di peluche che Xenia dona alla prima nipotina provocando la rabbia di Xeniuška ${ }^{[89]}$, ha trovato posto tra due testi che rappresentano le culture o le utopie che la storia e la memoria della famiglia hanno consegnato alla narratrice, facce inseparabili di un'identità che rifiuta di proporsi come monolitica e si vuole anzi dinamica, aperta, mutevole, ancorata al passato ma rivolta al futuro. Rivendicare la possibilità di essere insieme ebrei e comunisti, o di poter essere, come la nonna Xenia, rivoluzionari e sionisti, pur senza essere ebrei, significa liberarsi delle etichette, significa concepire la propria identità come qualcosa che si basa sui valori più che sulle appartenenze, pur senza voler cancellare le differenze che caratterizzano ciascun individuo: differenze plurime, innate o costruitesi nella storia, ma tutte necessarie a rendere più vario il mondo, se non diventano fonte di discriminazioni e intolleranze. La differenza, suggerisce la Sereni, pur se difficile da vivere e spesso scomoda, rappresenta sempre una ricchezza.

europei, pagherà con la vita la sua missione. Emilio, invece, abbraccerà insieme all'ideale sionistico l'ortodossia religiosa. Ma l'adesione successiva al comunismo lo indurrà a ripudiare, insieme al sionismo, anche il legame con il fratello e con gli altri familiari emigrati in Palestina.

[87] Sereni, C., Il gioco dei regni, op. cit., p. 194.

[88] Ivi, pp. 194-195.

[89] A p. 232 la Sereni scrive: "Xenia ripartì prima del previsto, vinta. [...] ed ogni cosa di nuovo era al suo posto. Tranne Miška che la bimba volle ad ogni costo [...] Esasperata da quel capriccio Xeniuška le concesse infine di giocare con l'orsacchiotto: ma dopo averlo percosso e percosso con il battipanni, perché se ne andasse via ogni traccia di passato". 
Adesso una parte del lavoro è finita: tanti fantasmi sono diventati in buona misura persone prima, e personaggi poi. Le fotografie di cui quasi ossessivamente ho costellato la mia stanza ora hanno un senso naturale di testimonianza, e non sono più scatole ostinatamente chiuse: c'è un ordine, benché lo sappia precario e suscettibile di modificazioni infinite.

Chi cambia ininterrottamente di posizione, da uno scaffale all'altro, dalla mia stanza a quella di mio figlio, è l'orso Miška, in questo momento pazientemente accoccolato nella libreria accanto a me fra un Bereshit rabbà e una Storia del Partito Comunista Italiano. Ma l'emozione che mi trasmette non muta, e sospetto che custodisca ancora molte eredità, nella sua pancia spelacchiata e consunta: che a premerla nel modo giusto, con attenzione e memoria, forse tuttora è capace di suonare ${ }^{[00]}$.

\section{BIBLIOGRAFIA}

ANDRÉ, M. C., 2001, Chicanas and Latin American Women Writers. Exploring the Realm of the Kitchen as a Self-Empowering Site, The Edwin Meller Press, New York.

CAPUTO, I., LEPRI, L., (a cura di), 1995, Conversazioni di fine secolo, La Tartaruga, Milano.

GIOVANARDI, S., 1997, Verso la forma romanzo: prospettive narrative di Clara Sereni, Edizioni minimum fax, Roma.

LA PORTA, F., 1999, La nuova narrativa italiana. Travestimenti e stili di fine secolo, Bollati Boringhieri, Torino.

MENOZZI, G., 1994, Food and Subjectivity in Clara Sereni's Casalinghitudine, in Italica, n.71, 217-227.

MUSARRA SCHRÖDER, U., 1989, Narciso allo specchio. Il romanzo moderno in prima persona, Bulzoni, Roma.

SERENI, E., SERENI, E., 2000, Politica e utopia. Lettere 1926-1943, La Nuova Italia, Torino.

SERENI, C., 1998, Taccuino di un'ultimista, Feltrinelli, Milano.

SERENI, C., 2007, Casalinghitudine, Biblioteca Universale Rizzoli (BUR), Milano.

SERENI, C., 2007, Il gioco dei regni, Biblioteca Universale Rizzoli (BUR), Milano.

SPLEEMAN, R., JANSEN, M., GAIGA, S., (a cura di), 2007, Scrittori italiani di origine ebrea ieri e oggi: un approccio generazionale, Italianistica Ultraiectina 2, Utrecht.

YEHOSHUA, A., 2001, Ebreo, israeliano, sionista: concetti da precisare, Edizioni e/o, Roma.

VAN DEN BOSSCHE, B., BASTIAENSEN, M., SALVADORI LONERGAN, C., (a cura di), 1998, Soavi sapori della cultura italiana, Franco Casati, Firenze.

[90] Sereni, C., Il gioco dei regni, op. cit., p. 425. 


\section{ŽENSKI SVIJET, DOM I OBITELJ U DJELIMA CLARE SERENI}

Clara Sereni rođena je u Rimu 1946. godine. Potječe iz poznate talijanske židovske obitelji. Otac, Emilio Sereni, bio je jedan od vodećih ljudi talijanskoga antifašističkog pokreta i član komunističke partije Italije nakon Drugoga svjetskog rata. Članak obrađuje neke od karakteristika pripovjedačkoga opusa Clare Sereni, s posebnim naglaskom na djela Casalinghitudine (1987.) i Il gioco dei regni (1993.). Djelo Taccuino di un'ultimista (1998.) polazište je za prikaz autoričina života i djela te ujedno dnevnik u kojem ona opisuje svoj politički angažman i djelovanje u organizacijama koje se bave socijalnom i humanitarnom djelatnošću i potrebama osoba s invaliditetom. U romanu Il gioco dei regni autorica na temelju prikupljenih dokumenta i osobnih sjećanja, kao i sjećanja drugih članova obitelji, rekonstruira svoje korijene, složenu prošlost njezine obitelji i svoj odnos s ocem, koji je imao odlučujući utjecaj na njezin životni put. Jednaku tematiku obrađuje i djelo Casalinghitudine, gdje hrana i pripremanje jela bude kod Clare Sereni razna sjećanja.

Ključne riječi: sjećanja, povijest, hrana, kulinarski recepti, obitelj, utopija

\section{FEMALE WORLD, DOMESTIC SPACE AND FAMILY IN CLARA SERENI'S WORKS}

Italian writer Clara Sereni was born in Rome (1946) and now lives in Perugia, where she served as Deputy Mayor of the city from 1995 to 1997 . Her father, Emilio Sereni, was one of the leading figures in the Italian antifascist movement and in the communist party. Her literary career began with the appearance of Sigma Epsilon (1974), but her masterpieces are Casalinghitudine (1987) and Il gioco dei regni (1993). As it can be seen in Casalinghitudine and in Il gioco dei regni, her writing allows for more nuanced representation of subjectivities, giving space for dreams, utopias, contradictions, and courage within the constraints of private space - such as the house - and public spaces and institutions - such as the political party, public office and the like. Casalinghitudine is the testimony of Sereni's striving to define women's language and writing. In this work domestic space and time of food preparation with various rituals represent the ideal location of memory, while the house is a dwelling and network of emotions that hides the mystery of the past. Il gioco dei regni retraces the history of three generations from the beginning of the twentieth century, and touches on all the emotions and contradictions, as well as utopias, of its characters, who are caught in their attempt to find balance sometimes unsuccessfully - between their sense of duty and responsibility towards their ideals on the one hand, and the reality of their present condition, on the other.

Key words: memory, history, food, recipe, family, utopia 